\title{
Extraction Experiments Using Commercially Available Spices
}

\author{
Armando M. Guidote Jr. ${ }^{*}$, Maricar O. Ribo-Ramos ${ }^{1,2}$ and Dante Gilbert L. De Leon ${ }^{2}$ \\ ${ }^{1}$ Department of Chemistry \\ School of Science and Engineering, Loyola Schools \\ Ateneo de Manila University \\ Loyola Heights, Quezon City Philippines 1108 \\ ${ }^{2}$ Science Area, \\ Miriam College High School, \\ Loyola Heights, Quezon City, Philippines 1108
}

\begin{abstract}
Safe and cost-effective experiments were designed for high school students to enhance their laboratory skills and knowledge on polarity and separation of mixtures. Annatto, Spanish paprika and turmeric were extracted yielding red-orange, light red-orange and yellow color, respectively. These were combined with a blue aqueous solution made from food color resulting to green mixtures. These were all extracted with kerosene or oil/kerosene. The non-polar solutes for each mixture combined with the kerosene or oil/kerosene layer, making the polar phase blue again. Students gained a better understanding of the concepts as shown in their laboratory report and test results
\end{abstract}

Keywords: high school, solubility, separation, annatto, Spanish paprika, turmeric

\section{INTRODUCTION}

The polarity of substances is a very abstract concept and students have a hard time understanding it unless a demonstration or a laboratory activity is given. There are laboratory activities that show the polarity of substances using the solubility of common substances like salt in various solvents, e.g., water to demonstrate the concept "like dissolves like" while other laboratory activities or demonstrations on polarity used colored compounds or colors extracted from plants [1-3].

There are also procedures on separating mixtures that involve the combination of different substances to form a mixture and then students separate the mixtures made using various separation techniques.

Here we present several extraction experiments that use mixtures that are safe and are easily found in the community namely annatto, Spanish paprika and turmeric. These spices were extracted using various solvents that are 
available from the supermarket and hardware store.

Annatto seeds are locally known as achuete and are used to color kare-kare and other Filipino dishes. The seeds are usually extracted by either using hot water to produce that reddish yellow to orange color or fried in vegetable oil or animal fats to achieve that golden yellow color for meat and several dishes. Annatto has the components bixin and norbixin whose structures are shown in Figure 1.

Paprika, an orange-red oil-soluble derived from red peppers (Capsicum annuum) is used for food, cosmetics, and pharmaceutical industries, replacing synthetic colorants because of paprika's nontoxic property [4]. It has $\beta$ carotene, zeanthin, violaxanthin and $\beta$ cryptoxanthin. Their chemical structures are shown in Figure 2.

Bixin, norbixin, $\beta$-Carotene, zeaxanthin, violaxanthin amd $\beta$-cryptoxanthin are carotenoids with characteristic conjugated double bonds responsible for the colors of these compounds. The color yellow is produced with a minimum of seven conjugated double bonds and its increase results to a shift of the major absorption bands due to the longer wavelengths and the hue becomes red [5].

Turmeric on the other hand is used for coloring textile and food. It is made-up of three curcuminoids: curcumin, demethoxycurcumin and bisdemethoxycurcumin [6]. The cucurminoid structure and its derivatives are shown in Figure 3

The objective of these experiments is to extract the colored components of the three spices and to show that they can be separated when combined with other mixtures to illustrate polarity of substances. Other than these, it also aims to provide chemistry teachers with an activity to enhance their lessons on polarity, solubility and/or separation of mixtures, and likewise, to help students understand any or all of these concepts for better appreciation of chemistry lessons.

\section{METHODOLOGY}

\section{Reagents}

Solvents (vegetable oil, kerosene, 95\% ethyl alcohol, 70\% isopropyl alcohol), Blue stock solution ( 1 drop of bright blue McCormick in $200 \mathrm{~mL}$ of distilled water). Annatto seeds, Spanish paprika and turmeric were bought from the supermarket.

\section{Extraction}

Annatto seed. One gram of whole annatto seeds was extracted using $10 \mathrm{~mL}$ of $70 \%$ isopropyl alcohol. This was stirred for 1 minute and filtered. A $20 \mathrm{~mL}$ aliquot of the blue stock solution was added to $1 \mathrm{~mL}$ of the annatto extract and stirred. The resulting mixture was placed in a test tube together with $10 \mathrm{~mL}$ of vegetable oil and $10 \mathrm{~mL}$ of kerosene. This was mixed vigorously for 1 minute and afterwards allowed to settle.

Spanish paprika. The paprika extract was prepared by combining $0.1 \mathrm{~g}$ of ground paprika in $50 \mathrm{~mL}$ of $95 \%$ ethanol. This was mixed well using a stirring rod and the solid was separated from the liquid through gravity filtration. A 10 $\mathrm{mL}$ aliquot of the blue stock solution was combined with $5 \mathrm{~mL}$ of the paprika extract. The mixture was then placed in a test tube together with $20 \mathrm{~mL}$ of kerosene. This was then covered with a cork and was mixed for a minute and allowed to stand.

Turmeric. A $0.1 \mathrm{~g}$ of powdered turmeric was extracted with $50 \mathrm{~mL}$ of $95 \%$ ethanol. This was stirred, filtered and $2 \mathrm{~mL}$ of this solution was mixed with $20 \mathrm{~mL}$ of the blue stock solution. The resulting mixture was combined with $20 \mathrm{~mL}$ of kerosene by shaking for 1 minute and was allowed to stand.

\section{Procedure for Evaluation of Activity}

Selected students from various public and private schools in Metro Manila attending the Chem Camp offered by the Ateneo de Manila University were given the laboratory activity for $1 \frac{1}{2}$ hours including pre-test and post test. They have two classes. One class performed the activity and the other did not. They both 
<smiles></smiles>

Fig. 1. Structures of the components of Annatto: bixin and norbixin.<smiles>CC1=C(/C=C/C(C)=C/C=C/C(C)=C/C=C/C=C(C)/C=C/C=C(C)/C=C/C2=C(C)CCCC2(C)C)C(C)(C)CCC1</smiles>

$\beta$-carotene<smiles>CC(/C=C/C=C(C)/C=C/C12OC1(C)CC(O)CC2(C)C)=C\C=C\C=C\C(C)=C\C=C\C(C)=C\C=C\C1(C)CC(O)CC(C)(C)C1</smiles><smiles>CC1=C(/C=C/C=C(C)/C=C/C=C(C)/C(C)=C/C=C/C=C(C)/C=C/C=C(C)/C=C/C2=C(C)CC(O)CC2(C)C)C(C)(C)CC(O)C1</smiles>

violaxanthin<smiles>CC1=C(/C=C/C(C)=C/C=C/C(C)=C/C=C/C=C(C)/C=C/C=C(C)/C=C/C2=C(C)CC(O)CC2(C)C)C(C)(C)CCC1</smiles>

$\beta$-cryptoxanthin

Fig. 2. Structures of the components of Paprika: $\beta$-carotene, zeanthin, violaxanthin and $\beta$-cryptoxanthin.<smiles>[R2]c1cc(/C=C/C(=O)CC(=O)/C=C/c2ccc(OC)c([R])c2)ccc1O</smiles>

Fig. 3. Structures of the components of tumeric: curcumin, demethoxycurcumin and bisdemethoxycurcumin. 
answered the pre-test and post test with the same time interval.

\section{Statistical treatment}

The $t$ test was used to evaluate the scores of the pre-test and post test of the two groups. The $t$ test for independent samples was used for comparing the results of the pre-test and post test of the two groups, and the $t$ test for the paired samples was used for comparing the difference in the results of the pre-test and post test of each group.

\section{RESULTS AND DISCUSSION}

After performing the experiment, the results are shown in Figures 4-6.

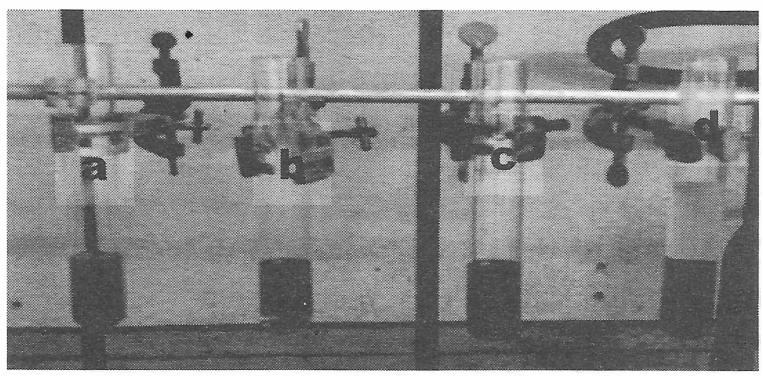

Fig. 4. After extracting annatto seeds with isopropyl alcohol, a red-orange mixture (a) was produced. When the blue solution(b) is combined with the extract, a green solution was achieved (c). When kerosene and oil were added to it, it was shaken to separate the components resulting to a blue to blue-green - colored aqueous solution while the upper portion became dark yellow (d).

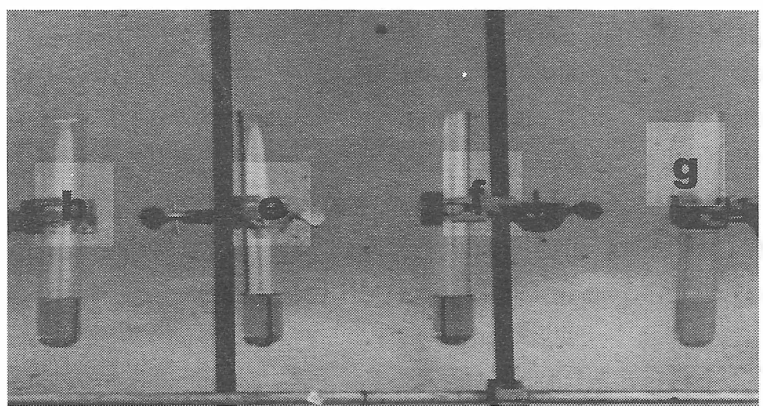

Fig. 5. After extracting paprika with ethanol, a light red-orange color was produced (e). The blue stock solution (b) was combined with the paprika extract and produced a light sea green color (f). When this solution was mixed with kerosene by vigorous shaking, a yellow upper solution and a lower blue aqueous solution were achieved ( $g$ ).

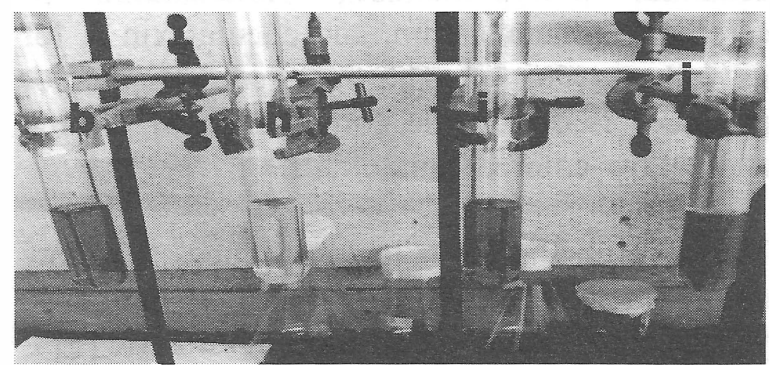

Fig. 6. After extracting turmeric with ethanol, a yellow color was produced (h). When the blue solution (b) was combined with the extract, a green solution was produced (i). After adding kerosene and shaking it, two phases were produced: the blue aqueous lower phase and the upper yellow solution that had combined with kerosene (j).

The different substances used to make food color were used to demonstrate the polarity of substances. Colored components of annatto seeds, turmeric powder and paprika were extracted using various solvents.

In this experiment, 70\% isopropanol and 95\% ethanol were used in extracting colored components from the spices in as much as the use of water results to poor extraction of colored components. The use of $70 \%$ isopropanol or $95 \%$ ethanol results in the extraction of moderately polar and non-polar substances.

For annatto seeds, a red-orange color is produced upon extracting it with $70 \%$ isopropyl alcohol because of the various compounds present in the seeds. When the mixture was separated by virtue of its polarity, the dark yellow-colored compounds combined with the upper kerosene/oil layer while the aqueous lower part is somehow blue to blue green in color. The 9' - cis - bixin, which is the ester of 9'-cis-norbixin is the component soluble in the non-polar layer [7] and is the one responsible for the yellow color. The water soluble dicarboxylic acid norbixin in annatto has a yellow to yellow - 
orange color and the reason behind the bluegreen lower layer.

Paprika has capsanthin and capsorubin as the main colorant responsible for its red color and zeanthin, $\beta$-cryptoxanthin, violaxanthin, $\beta$ carotene, antheraxanthin, and cucubitaxin $\mathrm{A}$ for its yellow color [8]. The light red-orange color produced is due to the combination of the red and yellow-colored compounds.

Turmeric on the other hand produces a yellow color when extracted with $95 \%$ ethanol. The yellow color is due to curcumin in turmeric [5].

The light yellow color of the non-polar layer after extraction is normal and can be explained by dilution. The polar layer may become light blue-green after extraction which can be explained by - incomplete extraction of the organic substances. The use of a solvent system, in this case kerosene, and a combination of vegetable oil and kerosene, was found to be necessary to hasten the separation. This can be used by the teacher to introduce the use of one solvent or a combination of solvents in extraction and to show why several extractions are necessary in a normal organic work-up. The vigorous shaking suffices to extract the different substances present in the three extracts and separate it from the blue substance of the food color that remain in the polar layer. When shaking is too vigorous an emulsion was produced.

\section{Student's performance}

Upon the computation of the pre-test using the $t$ test for independent samples, a $t$ value of 3.02 was obtained. The degree of freedom $(d f)$ is 67 but the $t$ distribution table has only 60 followed by 120 . Since the degree of freedom is not in the table, it is conventional to use the next smaller degree of freedom so in this case, a $d f$ of 60 was used [9]. The critical value in the column with a degree of freedom of 60 for a two-tailed test with $\alpha=0.01$ is 2.660 ; that is, $\mathrm{t}_{.01}(60)=2.660$. The computed value is higher than the critical value therefore there is a significant difference between the results of the pre-test of the two groups. The pre-test of the group that did not take the activity is higher as compared to the group that took the laboratory activity. When the post tests of the two groups were compared, there is no significant difference between their results. This is so since the computed value is 0.38 and the critical value is 2.660 using the $d f$ of 60 .

The $t$ test for dependent samples was used to compute for the difference in the result of the pre-test and post test of those who did not take the laboratory activity and also for the group that performed the laboratory activity. The computed value for the group that did not take the laboratory activity is 1.79 and the critical value with a $d f$ of 30 is 2.750 ; that is $\mathrm{t}_{.01}(30)=2.750$. This means that there is no significant difference between the results of the pre-test and post test of this group.

On the other hand, there is a significant difference between the pre-test and the post test of the group that took the laboratory activity. The computed $t$ value is 6.59 and the critical value for a $d f$ of 30 is 2.750 ; that is $t_{.01}(30)=$ 2.750. Since the computed value is higher than the critical value, this means that there is a significant difference between the post test mean as compared to the pre-test mean.

\section{CONCLUSION}

The extraction experiment presented in this paper is helpful in making students understand solubility and polarity of substances since the post test mean is significantly higher than the pre-test mean of the group that took the laboratory activity as compared to the group that did not perform the activity. The activity requires students to measure mass and volume properly so this can also be used to reinforce correct laboratory practices. This hands-on activity with some color changes will help students especially those who are kinesthetic and visual learners. Furthermore, the activity is easy to perform and the materials are relatively safe, inexpensive and easily available.

\section{REFERENCES}

1. de Leon, D.G.L., Guidote, A.M.Jr. J. Chem. Educ. 80, 436 (2003). 
2. Kitson, T.M. J. Chem.Educ. 80, 892 (2003).

3. Mihalick, J.E., Donnelly, K.M. J. Chem. Educ. 84, 96 (2007).

4. Guadayol, J. M.; Caixach, J.; Ribe, J.; Cabanas, J.; Rivera, J. J. Agric. Food Chem.; 45, 1868-1872 (1997).

5. Sikorski, Z.E. Chemical and Functional Properties of Food Components, 2nd ed. (CRC Press, 2002).
6. Jayaprakasha, G.K., Rao, L.J.M., Sakariah,K.K. J. Agric. Food Chem 50, $3668-3672$ (2002).

7. Scotter, M.J.,Wilson, L.A., Appleton, G.P., Castle, L. J. Agric. Food Chem 46, 1031 1038 (1998).

8. Hornero - Mendez, D, Minguez Mosquera, M.I J. Agric. Food Chem 49, $3584-3588$ (2001).

9. Spatz, C. Basic Statistics:Tales of Distribution, 8th ed. (Thomson Learning Inc., 2005). 